\title{
Design and Production Automation for Mass Customisation - An Initial Framework Proposal Evaluated in Engineering Education and SME Contexts
}

\author{
Leon Peter POOT ${ }^{1}$, Mehdi TARKIAN and Johan ÖLVANDER \\ Linköping University, Linköping, Sweden
}

\begin{abstract}
Maintaining high product quality while reducing cost is essential for mass-customised products, requiring continuous improvement of the product development process. To this end, design automation should be utilised in all stages of a product's develop process and lay the foundation for automation of repetitive tasks throughout the process from interaction with the customer to design and production in order to mitigate errors and minimise costs. In this paper, a design automation and production preparation framework is proposed that can facilitate automation from initial stages via $\mathrm{CAD}$ to production. Examples of the framework are shown in the shape of proof-of-concepts systems developed by master students in the context of a course in design automation at Linköping University. Included disciplines such as automated planning of robot assembly paths, CNC manufacturing files and production drawings are described, based on design automation, Knowledge-Based Engineering, and design optimisation. Additionally, variations of the framework are implemented at three SMEs, and the results thereof are presented. The proposed frameworks enable interaction and connection between the "softer", human centred, aspects of customer interaction within sales, with more traditional "harder" engineering disciplines in design and manufacturing.
\end{abstract}

Keywords. Design Automation, Mass Customisation, Product Configuration, Production Preparation, Transdisciplinary Engineering Education

\section{Introduction}

A competitive market combined with complex products and requirements has directed companies to manage complexity by continuously rationalising their product development process, aiming to minimise costs and increase quality. For this, automation is highly applicable in both design and manufacturing.

Mass customisation of complex products requires an engineer-to-order (ETO) approach, which allows for a high level of customisation by including the customer in early design stages [1]. Due to the high variation between products and the uniqueness of customer requirements, one-off parts are required in addition to standardised parts. Errors are more likely to occur during design and production due to the uniqueness of these parts than in the case of more traditionally mass-produced or even configured-toorder (CTO) products built solely from standardised parts. For mass-customised products

${ }^{1}$ Corresponding Author, Mail: leon.poot@liu.se. 
it is thus of utmost importance to mitigate errors in initial stages, as error-related costs incurred increase the further the development process progresses. Additionally, design and production of mass-customised products will incur increased time and costs as design- as well as production tasks must be repeated for alterations and variations. While the notion of cost varies depending on whom is affected and how, it is in this context primarily seen from the industry's point of view as a financial loss, either directly due to loss of materialistic or time resources or indirectly due to loss of reputation and customer trust in the company. Additional types of costs, ranging from environmental costs to the wellbeing of personnel, may however require attention during a product's development and remainder of its lifecycle.

Repetitive tasks are naturally fit for automation. Automation in manufacturing is well proven in industry and widely used within various fields, such as mechanical and electrical engineering. To accomplish mass customisation, the literature suggests different approaches such as modularisation [2], product families and platform design [3], which can be supported by methods such as Design Automation (DA) [4], product optimisation [5] and Knowledge-Based Engineering (KBE) [6].

When opting for DA the goal is to capture a wide range of the design rationale in a DA-tool. Advanced tools are able to produce both the geometric representation of the product i.e., the CAD model, and production data needed to automate the process from customer order to delivery. DA and KBE techniques are core to the development of product configurators, aimed at aiding product development in the (conceptual) design of product variants [7]. In combination with product optimisation, powerful design tools can be developed capable of sustaining mass customisation. A further aim with the introduction of DA configuration frameworks is to alleviate engineers from their noncreative and mundane tasks, and to intertwines actors from multiple disciplines including colleagues from both upstream and downstream of the product development process as well as with end-customers. This requires application of inter- or even transdisciplinary engineering methods to fully succeed. While successful examples of the implementation and use of DA in industry exist, companies continue to show a strong need for employees with skills and knowledge of how DA and KBE can be used together with product configurators to speed up their ETO-processes. Therefore, this paper describes how the proposed framework is implemented in a master's course, so that newly graduated engineers can bring this knowledge to industry. Additionally, implementations of the framework are discussed based on case studies in collaboration with three SMEs.

The remainder of this paper is structured as follows. After an initial state-of-the-art description, a general automation framework for design and production preparation of mass-customized products is proposed. Proof-of-concept implementations frameworks in the shape of results from a Masters' course in DA taught at Linköping University are described. Additionally, implementations of the framework are showcased from case studies in collaborations with Swedish industries before a discussion and concluding remarks.

\section{State of the Art}

According to Stokes [8], 80\% of (CAD) design tasks are routine-like and repetitive. These tasks are often time-costly and error-prone and are therefore suitably subject to DA [4]. Willner et. al. [9] confirm this, stating that "design automation based on integrating product configurators and CAD systems may result in a reduction of the 
engineering time by up to $90 \%$ ". One method of achieving DA is the application of KBE, applied in the form of knowledge-based systems (KBS), allowing existing knowledge to be utilised in order to create new products or product variants. Thus, repetitive tasks can be automated, supporting reuse of product knowledge as well as multidisciplinary optimisation throughout the entire design process [10].

Amadori \& Tarkian et al. [11] propose High Level CAD templates (HLCt) as a method for geometry automation with the aim of DA. Building on both morphological and topological geometry automation, the concept of HLCt is that each template contains attributes necessary in order to visualise the 3D geometry and provide contextual references for other templates. Additionally, the templates provide geometric and nongeometric attributes required for downstream tools such as simulation models, production files and Enterprise Resource Planning (ERP) and Product Lifecycle Management (PLM) integration. HLCt have been successfully applied in contexts such as rapid design of train carriages [12], robotic grippers [13] and spiral staircases [14], decreasing time, costs, and errors in design processes.

Highly customised designs can be produced by means of additive manufacturing due to the inherent geometrical freedom of the manufacturing process, as numerous methods supporting DA in design for additive manufacturing exist [15]. Mass-customised products are however often dependent on more traditional types of manufacturing due to functional, material, or other requirements. In these cases, production data such as drawings, $\mathrm{NC}$ machine code, and robot control programmes are required. CAM software can be utilised to automatically produce the $\mathrm{CNC}$ code needed to manufacture the virtual model in a physical setting. While such CAM systems are meant to be plug-and-play for CNC code generation, the reality is that extensive manual operations continue to be required. Therefore, works such as [16] are presented to fill the need reported in the industry. This is the case for many process planning systems, where lack of flexibility in handling varying inputs hinders complete automation [17].

The inclusion of sales in the engineering design process requires designers of a DA framework to consider the human elements involved and ease transdisciplinary communication to fully understand "soft" customer requirements, in addition to "hard" technical requirements, and thus solve an ill-defined problem. This requires not only technological integration but a common language between disciplines to break communicational barriers. [18]. This integration of disciplines within a large process requires a transdisciplinary engineering approach [19]. The education in engineering master programmes at Linköping University is conducted based on the educational framework CDIO (Conceive-Design-Implement-Operate) [20] and builds on a combination of disciplinary and interdisciplinary courses, with project courses looking towards transdisciplinary inclusion of human-focused aspects in engineering. Key points for transdisciplinary approaches include ill-defined and open-ended problems relevant for both academia and industrial practitioners [19]. The course presented in this paper attempts to addresses these issues in a project-based learning environment.

\section{Proposed Framework}

With the aim of achieving DA throughout the design process from conceptual design stages to production preparation, a framework incorporating HLCt-powered CAD configurators and auxiliary production preparation automation modules is proposed. The nature of such modules depends entirely on the production method these aim to support 
but will inevitably build on data introduced in earlier development stages, included in the templates with which the entire product model is built.

The DA implemented is based on the HLCt principle as outlined by [11]. Following chapters describe the additional improvements added to the principles of HLCt in order to enable automation of downstream models. HLCt allows the design space to be divided into smaller building blocks that can be easily modified, added, and removed. The addition of downstream logic does not affect existing design logic and can in itself be modified at any point in time without upstream complications, a strategy preferrable from a maintenance point-of-view.

Figure 1 illustrates the proposed framework including DA activities and examples of product preparation automation modules. To the left, a visualisation of the DA facet of the framework, where a CAD configurator is utilised to configure a customised product resulting in a configured model, based on user input. The CAD Configurator builds the model from a library of HLCt building blocks. Logic concerning the compatibility and required contextual and parametric inputs of the HLCt is drawn from a knowledgebase, which is compiled from the entire library beforehand. To the right of Figure 1, a set of production preparation automation methods, where information embedded in the model drawn from the HLCt in previous steps is utilised to automatically generate production documents and data, illustrated by the "Drawing Module", "Layout Optimisation" and "Product Simulation". Naturally, in a generic case the production preparation automation will include many more modules depending on the product and its manufacturing. In the forthcoming chapters, however, a subset of these models is described for illustrative purposes.

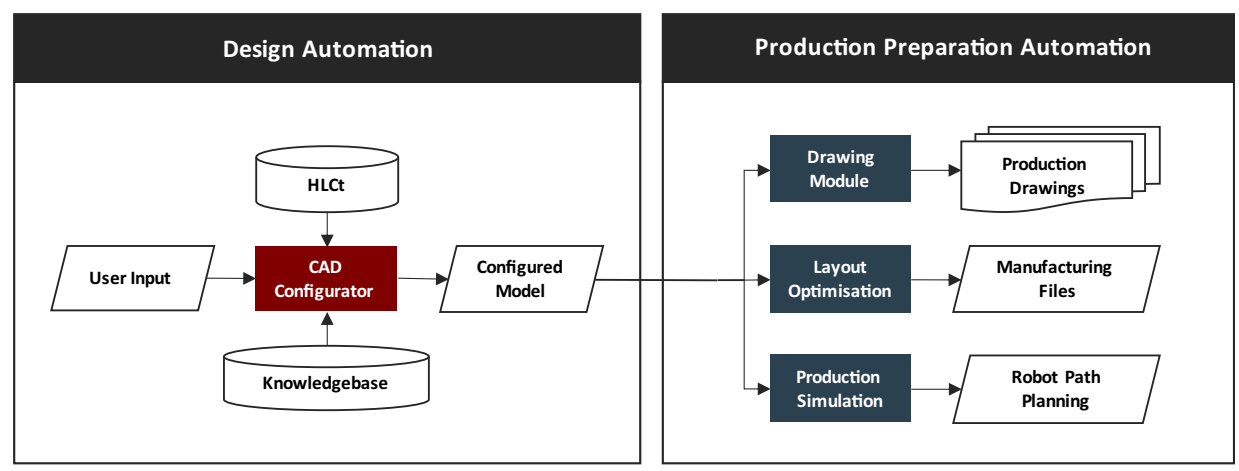

Figure 1. Proposed framework including Design Automation and Product Preparation Automation

\subsection{Design Automation and Product Configuration}

The framework builds on input models, modelled by the user from scratch or based on imported shapes, such as the resulting surfaces from a 3D scan. The user input will form the base on which HLCt are instantiated through the CAD configurator, thereby creating the detailed model required for the product development process to advance. 
HLCt templates include the parametrisation and contextual links required for both morphological and topological adaptation of the template geometry within the context into which it is instantiated. Information required for production preparation of the final product is embedded in the HLCt templates, the nature of which depending on the type of feature the template contains and the method of manufacturing/production it requires. As the detailed features are instantiated from the templates, a complete blueprint is formed from which production data can be generated.

\subsection{Production Preparation Automation}

Often encountered production data ranges from traditional formats such as production drawings for both manufacturing and assembly to machine-specific CNC code. Creation of production drawings continue to cost large amounts of money, up to a third of total engineering costs according to the US Department of Defense, with $60 \%$ of the drawings containing errors and inconsistencies [21]. Due to the repetitive nature of included tasks and all required data being readily available in CAD models, these are prime candidates for automation. Similarly, manufacturing data can be extracted based on the geometry of the CAD model and encoded to fit the application in question.

The proposed framework illustrates modules for generation of drawings and path files for $\mathrm{CNC}$ manufacturing as $2 \mathrm{D}$ representations, automatically recognised by the machine's operating system or line-by-line generated code. In the CAD environment, the drafting API is utilised to this end similarly to the generation of production drawings, albeit with the exclusion of humanly legible annotations. Production-specific datum geometry is included in the HLCt, instantiated within the 3D model. From these, (sectional) 2D views can be generated ready to be included in a drafting document and positioned based information included in the template. Positioning of multiple parts to be manufactured from the same specimen of material, requires an experienced user or optimisation algorithms in order to minimise manufacturing time and material costs. This can be achieved by optimising the position and rotation of each part upon a common plane, with a goal function towards a minimum area of the resulting bounding box of all parts constrained by the material's and/or machine's limitations.

In order to define a robot assembly programme a set of operations need to be defined in a robot program. Depending on the geometry of the parts of a product (represented in the CAD models) the number, size, position, and rotation of these operations will be different. In this study DELMIA is utilised as the robot offline programming software. By utilising HLCt, product assemblies are never pre-configured, yet information needed to automatically setup any given configuration can be embedded. All HLCt contain contextual information concerning their compatibility and geometric interaction with each other. The same technique is used to automatically generate, i.e., configure, the production cell. Every geometry is predefined in a template, including the information necessary to be instantiated correctly in context. This includes robots, components to be processed and any additional tools that may be required. Once the production cell has been populated, the robot paths can be generated.

For any given production process, tasks can be defined with Robot Operations in DELMIA, requiring user-defined start, end, and way points. These are defined as coordinate systems to describe the position and orientation of the robot gripper in various steps of the operation. Options include operations such as Motion, Delay, Pick, or Place. Definitions of operation-specific objects and parameters are embedded in the HLCt, rendering complete automation of robot path generation possible. 


\section{Student Course Results}

The framework is put into context in the course TMKU01 Design Automation of Customized Products taught at Linköping University as part of the master's programmes in Mechanical Engineering and Design and Product Development. Conducted towards the end of the programmes, pre-requisite disciplinary knowledge includes parametric CAD modelling and DA as well as multidisciplinary design optimization. Intended learning outcomes include understanding of the correlation of digital models with reality, the use of customer data to drive development of customised products and the suitability of different digitalisation methods depending on the type of product. Run in project-form with the application or product to be chosen by the students, and thus without pre-defined answers, the course builds on open-ended problems. Projects are selected based on level of complexity and realism, in order to appropriately simulate industrial cases. Focus in the course lies on applying knowledge of multiple disciplines and learn through a practical example to combine these in a process from early customer and human centred interaction to detailed technical design and production.
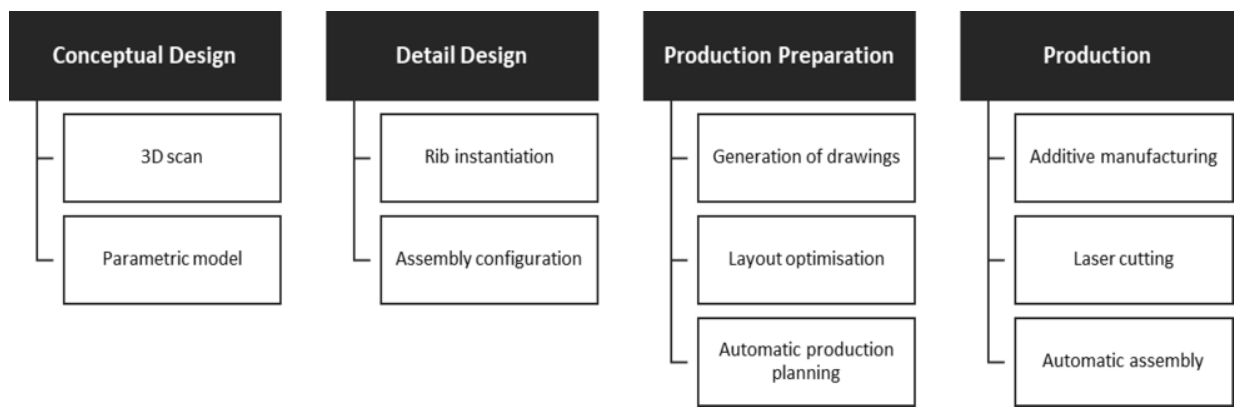

Figure 2. Automation tasks undertaken in the course Design Automation of Customized Products.

Students are required to design a product of their choice utilising DA, while allowing the product to be configurable and customisable. Scale models are produced using a CNC controlled laser-cutter machine and assembled using an industrial robot. The entire process from concept to assembly is simulated through steps incorporating conceptual design, detail design, production preparation and production, as shown in Figure 2. Between each step, DA is achieved through the use of KBE as described above. Hence the complete framework is simulated and tested in a simplified educational setting.

All modelling and drafting is undertaken in CATIA V5. Physical mock-ups are digitised by means of 3D scanning, and a parametric model of the conceptual shape is generated from the scan with HLCt. Detailed parts are instantiated from HLCt templates and adapted to the input model. In the case of for example an aerocraft, structural rib members are instantiated to fit the model as the entire assembled product model takes shape. With all required information included in the assembled model, production preparation steps are taken before a physical scale model is produced.

As part of the production preparation automation, students produce a framework capable of generating technical drawings for manufacturing and assembly based on the products configured using the CAD configurator. Based on vertices in the model measurements are added, while adhering to the commonly agreed upon rules for technical drawings. An example of generated drawing features can be seen in Figure 3.

Generation of manufacturing files is accomplished as described in previous sections utilising the drafting workbench. The result is essentially a bare technical drawing sans 
annotation, exported in the form of a vector image for input to the CNC controlled lasercutting machine functioning as the main manufacturing tool. Prior to generating robot paths, the layout of the parts to be manufactured within the material's limitations is optimised using the optimisation software ModeFRONTIER, which is directly linked to parameters in the CATIA model as well as an Excel worksheet functioning as graphical user interface and user data storage. Constraints are set in ModeFRONTIER based on the material's limitations read from user data. During optimisation, values of parameters controlling the parts' locations are controlled by the algorithm and custom functions incorporating CATIA API calls are executed for each iteration. These functions execute clash analyses within the CAD environment, of which the results are returned to the algorithm for evaluation. The optimisation algorithm utilised in this process is the genetic algorithm NSGA-II [23].

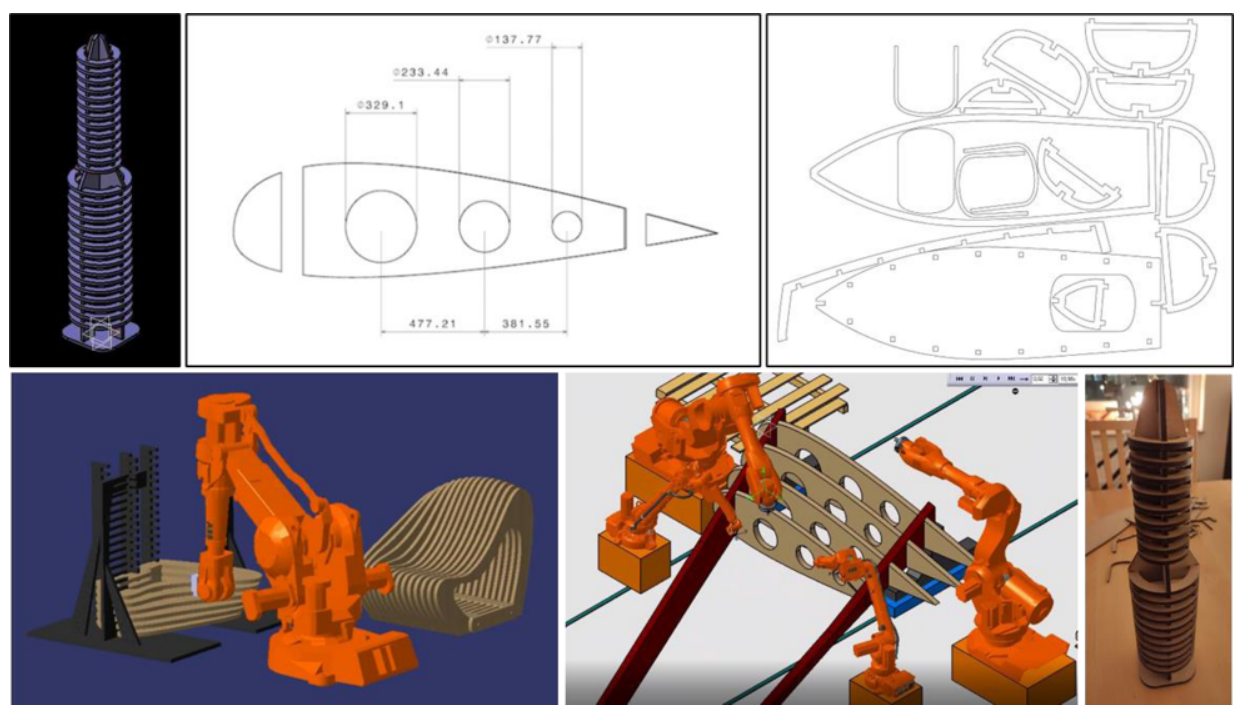

Figure 3. A selection of project results in various stages of the framework. From top left to bottom right: A configured model, generated drawing features, generated $\mathrm{CNC}$ paths, robot assembly in simulation, and an assembled scale model. Models depict, in order, a residential tower, structural members of an aerocraft's wing, structural members of a sailboat, and modular furniture.

The production preparation process for laser-cut parts is entirely automated and requires no user interaction other than specifying limitations and a 3D model of the product to be manufactured. The process takes, optimisation included, approximately one hour on a workstation running Windows 10 on a quadcore CPU at $3.5 \mathrm{GHz}$ with $16 \mathrm{~GB}$ RAM. Examples of layouts, CNC paths, and assembled scale model are shown in figure 3.

In order to fulfil the automation requirements, the framework includes a module for generation of robot paths based on positions rendered from the 3D model itself. Creation and simulation of paths is achieved in DELMIA utilising the production planning software's API. To perform a complete assembly of the product, a sequence of robot operations is generated as described in section 2.3. Generic functions for creation of operations and tags are required, which control the DELMIA functions within the CATIA environment through the API. The manner in which parts, robot(s), fixtures, and other manufacturing details are positioned is encoded specifically for each application. The benefit of this approach stems from the opportunity to alter the design of the 
product's morphology and topology in its entirety while still being able to utilise the existing robot planning script to regenerate the robot's offline code.

Figure 3 shows still images of automatically planned robot assembly simulations created during the course. Both images show the required models for robots, fixtures, and parts to be assembled. In this case structural rib members for sections of a park bench and rib structures of an aircraft wing. In both cases, the ribs shown are a direct result of the described DA and production preparation methods.

Knowledge transfer is confirmed throughout the project through continuously dialogue between students and teachers. Examination is conducted in a seminar setting where the project results are presented. Students are required to provide proof of adequate knowledge by discussing the suitability of different methods, and the impact their results could have on a product development process and personal involved therein in an industrial setting.

\section{Industrial Implementations}

The proposed framework demonstrated in the student course has been implemented in an industrial setting within the context of the research project e-FACTORY. Due to confidentiality agreements, the details of those implementations cannot be disclosed. However, a summary of their outcome is given below. Within the research project the proposed automation framework has been implemented at three companies, including a unique CAD-configurator for each, as well as product-specific HLCt and exclusive knowledgebases. In three distinctive settings various configurators were applied for three of the partner companies. The type of product and CAD and configuration software for each case can be seen in Table 1 .

Table 1. Company case details

\begin{tabular}{cccc}
\hline Company & Product & CAD Software & Configuration Software \\
\hline A & Industrial staircases & SolidWorks & XperDi CAD Configurator \\
B & Industrial staircases & Inventor & Developed in-house \\
C & Conveyor belts & SolidWorks & DriveWorks \\
\hline
\end{tabular}

Each company has been able to automate a large portion of previously manually undertaken repetitive $\mathrm{CAD}$ processes. By storing information for downstream tasks within configured models, all three companies were able to automatically generate 2Ddrawings and manufacturing files. Apart from time savings, the most appreciated advantage of configurator-based DA is the mitigation of errors in the process. To verify the effect of the developed frameworks, workshops and interviews have been conducted with engineers and salespersons at each company, with representatives stating that the implementation has meant huge time savings in administrative work on the models. Company $\mathrm{C}$ estimates that they can save up to $90 \%$ of administrative time from quotation via order registration, purchasing, and control of stock balance to technical documentation. Respondents have stated the expectancy that the framework will provide improved sales support and handling of existing customer relations, while being able to increase the number of quotes generated for new customers as online configurator are to be released. In this context, the configurators' ability to provide customers direct access to offers and drawings at any time is seen as a considerable advantage. 


\section{Discussion \& Conclusion}

A successful implementation of a DA framework enables interaction and connection between the "softer", human-centred, aspects of customer interaction within sales, with more traditional "harder" engineering disciplines in design and manufacturing. Inclusion of salespersons in the engineers' process, and vice versa, requires designers of a DA framework to consider the human elements involved and ease transdisciplinary communication. Education in transdisciplinary engineering is of utmost importance, with movements such as Industry 4.0 pushing towards greater integration between disciplines and industries' increasing focus on mass customisation. The course described is one step towards a wide-spread understanding of the importance and means of coupling deeply technical with surrounding disciplines.

Based on observations in relation to the introduction of the described framework in industrial settings, it is apparent that the industry is in need of versatile engineers capable of understanding both technical and human factors in the product development process. Results from the industrial implementations have both verified the need for and improved the described course. While this particular course merely ticks part of the CDIO checklist for complete TE education, it is its position in a master's programme that fosters its students' understanding of interdisciplinary methods by combining topics from several disciplinary courses and applying these in a simulated industrial setting, preparing the students for their eventual thesis projects and careers. To enhance the impact of the course's teachings and its link to reality, future course projects are to include close contact with industrial actors acting as product owners.

The framework proposed in this paper is highly dependent on the use of HLCt, being the building block and information carrier for the majority of the information required for design and production preparation, chosen due to the flexibility and maintainability it provides the user over centrally stored, hardcoded product knowledge.

While modern production methods relying on automation may lessen the requirement for traditional drawings during production, human-readable documentation will undoubtedly continue to be required for quality assessment, testing, maintenance, nonautomatable production methods and other human interactions with a product throughout its lifecycle. Provided the HLCT includes the appropriate information, any such documentation can be generated for mass-customised products from the configured model with specialised framework. Similarly, existing stand-alone programs such as process-specific $\mathrm{CAD} / \mathrm{CAM}$ software can be incorporated, requiring such software to be accessible through an API and flexible enough for true mass customisation, as the tool itself should not limit upstream design freedom. The framework may be implemented in different stages of the product development process, be it variant design of well-defined product families or conceptual design of individual parts or indeed entirely new product families, enabling the user to rapidly iterate through concepts and assess their feasibility.

A modular DA approach based on the concept of HLCt provides a flexible yet robust configuration process and enables automated production preparation by efficient reuse of knowledge. Implementation in the production of existing products in SME contexts has shown both the need for and potential effectiveness of the framework proposed, easing communication between disciplines. Initial results from both student projects and industrial cases call for further verification of the included production preparation methods as well as exploration of more advanced application in realistic settings and inclusion of additional engineering disciplines. 


\section{Acknowledgement}

The authors would like to thank Vinnova for funding this work as part of the research project e-FACTORY (ref. nr. 2018 01584), as well as the project members for their valuable input and advice. The hard work and feedback of all students in the Linköping University course TMKU01 during the fall of 2020 are greatly acknowledged.

\section{References}

[1] C. E. Levandowski, J. R. Jiao, and H. Johannesson, A two-stage model of adaptable product platform for engineering-to-order configuration design, J. Eng. Des., 2015, Vol. 26, No. 7-9, pp. 220-235.

[2] A. Ericsson and G. Erixon, Controlling Design Variants: Modular Product Platforms, Society of Manufacturing Engineers, Dearborn, 1999.

[3] J. Jiao, T. W. Simpson, and Z. Siddique, Product family design and platform-based product development: a state-of-the-art review, Journal of Intelligent Manufacturing, 2007, Vol. 18, No. 1, pp. 5-29.

[4] M. Tarkian, Design Automation for Multidisciplinary Optimization: A High Level CAD Template Approach, PhD dissertation, Linköping University Electronic Press, Linköping, 2012.

[5] J. Andersson, Multiobjective Optimization in Engineering Design: Applications to Fluid Power Systems, PhD dissertation, Linköpings universitet, Linköping, 2001.

[6] G. La Rocca and M. van Tooren, Knowledge based engineering to support complex product design, Advanced Engineering Informatics, 2012, Vol. 26, No. 2, pp. 157-158.

[7] A. Trentin, E. Perin, and C. Forza, Product Configurator Impact on Product Quality, Int. J. Prod. Econ., 2012, Vol. 135, No. 2, pp. 850-859.

[8] M. Stokes, Managing Engineering Knowledge: MOKA: Methodology for Knowledge Based Engineering Applications, Professional Eng. Pub., London, 2001.

[9] O. Willner, J. Gosling, and P. Schönsleben, Establishing a maturity model for design automation in salesdelivery processes of ETO products, Computers in Industry, 2016, Vol. 82, pp. 57-68.

[10] G. La Rocca, Knowledge based engineering: between AI and CAD. Review of a language based technology to support engineering design, Advanced Eng. Inform., 2012, Vol. 26, No. 2, pp. 159-179.

[11] K. Amadori, M. Tarkian, J. Ölvander, and P. Krus, Flexible and robust CAD models for design automation, Advanced Engineering Informatics, 2012, Vol. 26, No. 2, pp. 180-195.

[12] V. Gopinath, M. Tarkian, J. Ölvander, and W. Gaziza, Template driven conceptual design of high speed trains, Proc. of the ASME 2014 IDETC/CIE, Vol. 2A: 40 th Des. Autom. Conf., 2014.

[13] M. Honarpardaz, J. Ölvander, and M. Tarkian, Fast finger design automation for industrial robots, Robot. Auton. Syst, 2019, Vol. 113, pp. 120-131.

[14] L. P. Poot, C. Wehlin, M. Tarkian, and J. Ölvander, Integrating sales and design: applying CAD configurators in the product development process, Proc. Des. Soc. Des. Conf., 2020, Vol. 1, pp. 345-354.

[15] A. Wiberg, J. Persson, and J. Ölvander, Design for additive manufacturing - a review of available design methods and software, Rapid Prototyping J., 2019, Vol. 25, No. 6., pp. 1080-1094.

[16] J. Braumann and S. Brell-Cokcan, Parametric robot control: Integrated CAD/CAM for architectural design, Integr. Through Comput. - Proc. 31st Annu. Conf. Assoc. Comp. Aided Des. Arc. ACADIA, 2011.

[17] M. Al-wswasi, A. Ivanov, and H. Makatsoris, A survey on smart automated computer-aided process planning (ACAPP) techniques, Int. J. Adv. Manuf. Technol., 2018, Vol. 97, No. 1-4, pp. 809-832.

[18] M. Butt, A. Sharunova, M. Storga, Y. I. Khan, and A. J. Qureshi, Transdisciplinary Engineering Design Education: Ontology for a Generic Product Design Process, Procedia CIRP, 2018, Vol. 70, pp. 338-343.

[19] N. Wognum, C. Bil, F. Elgh, M. Peruzzini, J. Stjepandić, and W. J. C. Verhagen, Transdisciplinary systems engineering: Implications, challenges and research agenda, International Journal of Agile Systems and Management, 2019, Vol. 12, No. 1, pp. 58-89.

[20] E. F. Crawley, J. Malmqvist, S. Östlund, D. R. Brodeur, and K. Edström, Rethinking engineering education: The CDIO approach, second edition. Springer International Publishing, 2014.

[21] B. Boehm, J. Bayuk, A. Desmukh, R. Graybill, J. Lane, A. Levin, M. Azad, M. McGrath, A. Pyster, S. Tarchalski, R. Turner, and J. Wade, Systems 2020:Strategic Initiative. (report SERC-2010-TR-009), 2010.

[22] M. Honarpardaz, M. Tarkian, J. Ölvander, and X. Feng, Finger design automation for industrial robot grippers: A review, Rob. Auton. Syst., 2017, Vol. 87, pp. 104-119.

[23] K. Deb, S. Agrawal, A. Pratap, and T. Meyarivan, A fast elitist non-dominated sorting genetic algorithm for multi-objective optimization: NSGA-II, IEEE Trans. Evol. Comput., vol. 6, no. 2, pp. 182-197, 2002. 